\title{
Generation of expressed sequence tags for discovery of genes responsible for floral traits of Chrysanthemum morifolium by next-generation sequencing technology
}

Katsutomo Sasaki ${ }^{1 *}$, Nobutaka Mitsuda ${ }^{2}$, Kenji Nashima ${ }^{3,4}$, Kyutaro Kishimoto $^{1}$, Yuichi Katayose $^{5}$, Hiroyuki Kanamori ${ }^{5}$ and Akemi Ohmiya ${ }^{1}$

\begin{abstract}
Background: Chrysanthemum morifolium is one of the most economically valuable ornamental plants worldwide. Chrysanthemum is an allohexaploid plant with a large genome that is commercially propagated by vegetative reproduction. New cultivars with different floral traits, such as color, morphology, and scent, have been generated mainly by classical cross-breeding and mutation breeding. However, only limited genetic resources and their genome information are available for the generation of new floral traits.

Results: To obtain useful information about molecular bases for floral traits of chrysanthemums, we read expressed sequence tags (ESTs) of chrysanthemums by high-throughput sequencing using the 454 pyrosequencing technology. We constructed normalized cDNA libraries, consisting of full-length, 3'-UTR, and 5'-UTR cDNAs derived from various tissues of chrysanthemums. These libraries produced a total number of 3,772,677 high-quality reads, which were assembled into 213,204 contigs. By comparing the data obtained with those of full genome-sequenced species, we confirmed that our chrysanthemum contig set contained the majority of all expressed genes, which was sufficient for further molecular analysis in chrysanthemums.

Conclusion: We confirmed that our chrysanthemum EST set (contigs) contained a number of contigs that encoded transcription factors and enzymes involved in pigment and aroma compound metabolism that was comparable to that of other species. This information can serve as an informative resource for identifying genes involved in various biological processes in chrysanthemums. Moreover, the findings of our study will contribute to a better understanding of the floral characteristics of chrysanthemums including the myriad cultivars at the molecular level.
\end{abstract}

Keywords: Chrysanthemum morifolium, Expressed sequence tag, Next-generation sequencing technology, Transcription factor, Transcriptome

\section{Background}

Chrysanthemum (Chrysanthemum morifolium) is one of the most commercially important ornamental plants in the world. New chrysanthemum cultivars have been generated mainly by classical cross-breeding and mutation breeding [1-3].

\footnotetext{
* Correspondence: kattu@affrc.go.jp

${ }^{1}$ Institute of Vegetable and Floriculture Science, National Agriculture and Food Research Organization (NARO), 2-1 Fujimoto, Tsukuba, Ibaraki 305-0852, Japan

Full list of author information is available at the end of the article
}

Chrysanthemums are an allohexaploid $(2 \mathrm{n}=6 \mathrm{x}=54)$, with somatic chromosome numbers varying within the range of $2 n=47-63$, and have multiple loci because of a loss or gain of several chromosomes $[4,5]$. It is difficult to obtain S1 progeny because, similarly to many other plants that belong to the Asteraceae family, the sporophytic self-incompatibility of chrysanthemums [6] precludes selfing $[7,8]$. Therefore, for commercial production, chrysanthemum cultivars have been propagated asexually. In addition, the genome size of chrysanthemum cultivars is enormous $(12.381-24.802$ Gbp; 
http://www.etnobiofic.cat/gsad_v2/. Accessed Aug 23, 2017) when compared with those of other model plants such as Arabidopsis (Arabidopsis thaliana; 0.125 Gbp) [9] and rice (Oryza sativa L.; 0.389 Gbp) [10]. Furthermore, the chimerical structure should be considered in the analysis of ray-petal-color inheritance [11]. Due to these characteristics, obtaining a pure line that is ideal for genome and genetic analyses is difficult, and the progress of the inheritance analysis of ornamental traits is hindered.

Genetic analyses in model plants, such as Arabidopsis and snapdragon (Antirrhinum majus), and the reverse genetic analysis in many plant species have revealed that transcription factors (TFs) play important roles in the development of floral organs and floral traits. In the genome of Arabidopsis, 1717 TF loci exist (PlantTFDB v4.0; http://planttfdb.cbi.pku.edu.cn/index.php?sp=Ath, Accessed Aug 23, 2017) [12] which were classified into more than 50 families according to differences in their DNA binding domains [13]. TFs not only determine the floral organ identity (ABC model) [14-16] but also contribute to the development of floral traits, such as morphology, colors, scent, and floral architecture [17-19]. However, the functions of TFs that participate in the identification of floral organs and/or characterization of their ornamental traits in chrysanthemums have been poorly understood, because the information concerning their genomic sequences is scarce, and performing a genetic analysis is difficult.

Ray petal colors of ancestral chrysanthemums are limited to pink, yellow, and white, which derived from anthocyanins and/or carotenoids, and absence of these pigments, respectively [20,21]. Because flower color is a crucial ornamental trait, much effort has been devoted to the production of novel flower colors [2]. In the modern chrysanthemum cultivars, a wide range of flower colors, including purplish red, orange, red, and dark red, have been developed by increasing the range of pigment content or combinations of these two pigments. Recently, green-flowered cultivars containing chlorophyll in their petals have become popular. However, knowledge of the molecular basis of the control of metabolic pathways of these pigments in the flowers of chrysanthemums is still limited.

Floral scent is another factor that affects the commercial value of chrysanthemum cultivars. Nearly 200 volatile compounds have been identified in the flowers of chrysanthemum cultivars. The main compounds are monoterpenes and oxygenated monoterpenes, such as camphor, $\alpha$-pinene, chrysanthenone, safranal, myrcene, and eucalyptol [22]. These compounds are not only responsible for the floral scent characteristics of chrysanthemums, but they also provide antioxidant activity in the use of chrysanthemums as herbs [23]. Importantly, an improvement of floral scent properties would be necessary to increase the commercial value of chrysanthemum cultivars. However, the molecular mechanism that regulates such a complex mixture of volatile compounds in chrysanthemums is not elucidated.

Expressed sequence tag (EST) sequencing is important for conducting functional analysis, especially in horticultural plants whose genome information is lacking. Due to the large genome and high genome polyploidy, the complete genome sequence of chrysanthemums is difficult to read. In this study, we performed a large-scale transcriptome analysis using a next-generation sequencer in chrysanthemums and obtained a considerable amount of EST data and more than 200,000 contigs. The contigs contained a series of genes related to pigment and aroma compound metabolism, i.e., TF families which have been previously reported in Arabidopsis. This work will make a significant contribution as a reference for future studies toward a genetic, biochemical, and molecular understanding of the floral traits of chrysanthemums.

\section{Results and discussion}

\section{EST sequencing and assembly}

For the EST sequencing, we used the chrysanthemum cultivar 'Sei-Marine' (Fig. 1), which has a single-type flower with two types of florets, disk florets, and ray florets. We prepared four independent cDNA libraries to maximize the range of expression transcript diversity (Table 1). These cDNA libraries were produced by RNA mixtures extracted from several vegetative and floral organs at various developmental stages. Because chrysanthemum cultivars are mostly allohexaploid with loss or

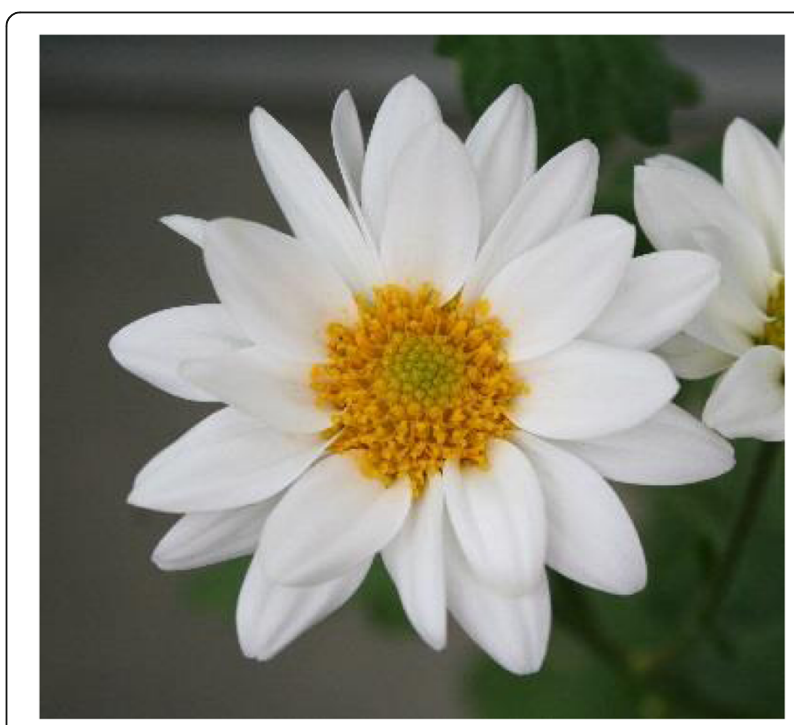

Fig. 1 A photograph of a flower of the Chrysanthemum morifolium cultivar 'Sei-Marine' used in this study 
Table 1 Summary of chrysanthemum transcript data

\begin{tabular}{|c|c|c|c|c|c|}
\hline Library & $1 s t$ & 2nd & $3 r d$ & 4th & Total \\
\hline Organs & Floral organs & Entire organs & Entire organs & Entire organs & \\
\hline Type of cDNA & full length & full length & 3' purified & 5' purified & - \\
\hline Total number of reads & $1302 k$ & $761 k$ & $1001 k$ & $708 k$ & $3773 k$ \\
\hline Average sequence length & $358 \mathrm{bp}$ & $585 \mathrm{bp}$ & $457 \mathrm{bp}$ & $470 \mathrm{bp}$ & - \\
\hline Total bases & $466 \mathrm{Mbp}$ & $446 \mathrm{Mbp}$ & $457 \mathrm{Mbp}$ & $332 \mathrm{Mbp}$ & $1702 \mathrm{Mb}$ \\
\hline
\end{tabular}

gain of several chromosomes [4,5] and their genomes are allopolyploid, the existence of many singlenucleotide polymorphisms (SNPs) was estimated even though the genomic/RNA sequences were derived from only one cultivar. We, therefore, chose to employ the GS FLX 454 system for the EST sequencing, which can read relatively longer DNA sequences, because short-read sequences were supposed to be inadequate for assembling the chrysanthemum ESTs containing abundant SNPs. The 454 sequencing process was performed after the normalization of the following four cDNA libraries. The first cDNA library was prepared by RNAs derived only from floral organs, providing a total number of $1302 \mathrm{k}$ reads. The second, third, and fourth cDNA libraries were prepared by RNAs derived from various aerial organs of the chrysanthemum, including flowers, leaves, stems, and the total numbers of these reads were $761 \mathrm{k}, 1001 \mathrm{k}$, and $708 \mathrm{k}$, respectively. In particular, the third and fourth cDNA libraries were purified as $3^{\prime}$ - and $5^{\prime}$-cDNA libraries to increase the available sequences from the 3 ' and $5^{\prime}$ ends of the transcripts. A total of $3773 \mathrm{k}$ reads from these four cDNA libraries were obtained from the 454 sequencing run. The average sequence lengths of these four cDNA libraries were $358 \mathrm{bp}, 585 \mathrm{bp}, 457 \mathrm{bp}$, and $470 \mathrm{bp}$, respectively. Because the cDNA sequences were sufficiently long for the 454 sequencing system, the generated cDNA libraries were supposed to be of good quality. The total numbers of the bases of these cDNA libraries were $466 \mathrm{Mbp}, 446 \mathrm{Mbp}, 457 \mathrm{Mbp}$, and 332 $\mathrm{Mbp}$, respectively, and a total of $1702 \mathrm{Mbp}$ bases were sequenced using the 454 sequencing method.

The $3773 \mathrm{k}$ sequences $(3,772,677$ reads; Table 2) were assembled after trimming and filtering processes. The 3 ' and $5^{\prime}$ adaptor sequences, which were used for the construction of these cDNA libraries, were removed from

Table 2 Summary of filtering and assemblies of chrysanthemum transcriptome data

\begin{tabular}{ll}
\hline Total Reads & $3,772,677$ \\
\hline Available reads evaluated after filtering & $3,573,676$ \\
Invalid reads for assembling & 324,723 \\
Singlets that were not used for construction of contigs & 5367 \\
Reads used for construction of contigs & $3,243,586$ \\
Contigs & 213,204 \\
\hline
\end{tabular}

the 3,772,677 sequences. Next, the polyT signals on the 5 '-terminal side of the reading sequences were removed. In addition, the polyA signals on the 3 '-terminal side of the sequences were also eliminated. The read sequences whose lengths were less than $40 \mathrm{bp}$ after these trimming processes, were excluded from the cDNA assembly, and thus a total of 3,573,676 sequences remained for the assembly. Next, the assembling was performed with MIRA (version 3.4.0) [24] using these 3,573,676 read sequences. Furthermore, the read sequences that matched to a huge larger of other reading sequences and sequences containing short repeats were excluded. Consequently, $3,243,586$ reading sequences were used for the assembly, and 213,204 contigs were finally obtained.

\section{Functional annotation}

The 213,204 contigs were annotated using the Arabidopsis protein data set (TAIR10; https://www.arabidopsis.org/ portals/genAnnotation/gene_structural_annotation/annotation_data.jsp; Accessed Aug 23, 2017) from the Arabidopsis Information Resource (TAIR) (https:// www.arabidopsis.org/; Accessed Aug 23, 2017) through the BLASTX program using an E-value cutoff of 1e-5 according to the classification of Gene Ontology (GO). As a result, $61 \%$ of the contigs produced top-hits to 16,740 loci of Arabidopsis in total. Because only 15,297 loci were tophit when all rice genes were employed as BLASTX queries to all Arabidopsis proteins, we considered that our contig set covered the majority of all genes expressed in chrysanthemums. The GO Slim classification of the Arabidopsis gene models was applied to estimate the functional role of each contig. The functional classifications of the contigs that matched Arabidopsis genes within each GO term are shown in Fig. 2. In the molecular function category, "nucleotide binding (9.2\%)," "hydrolase activity (8.0\%)," "transferase activity (7.6\%)," "protein binding (8.6\%)," and "DNA or RNA binding (6.9\%)" are highly represented terms. In the biological process category, "protein metabolism (7.4\%)," "response to stress (7.4\%)," "response to abiotic or biotic stimulus (7.0\%)," "cell organization and biogenesis (5.9\%)," "developmental process (5.5\%)," and "transport (5.8\%)" are highly represented terms. In the cellular component category, "nucleus (15.4\%)" is the most highly represented, and "chloroplast (9.3\%)" and "plasma membrane (7.1\%)" are highly represented terms. The 


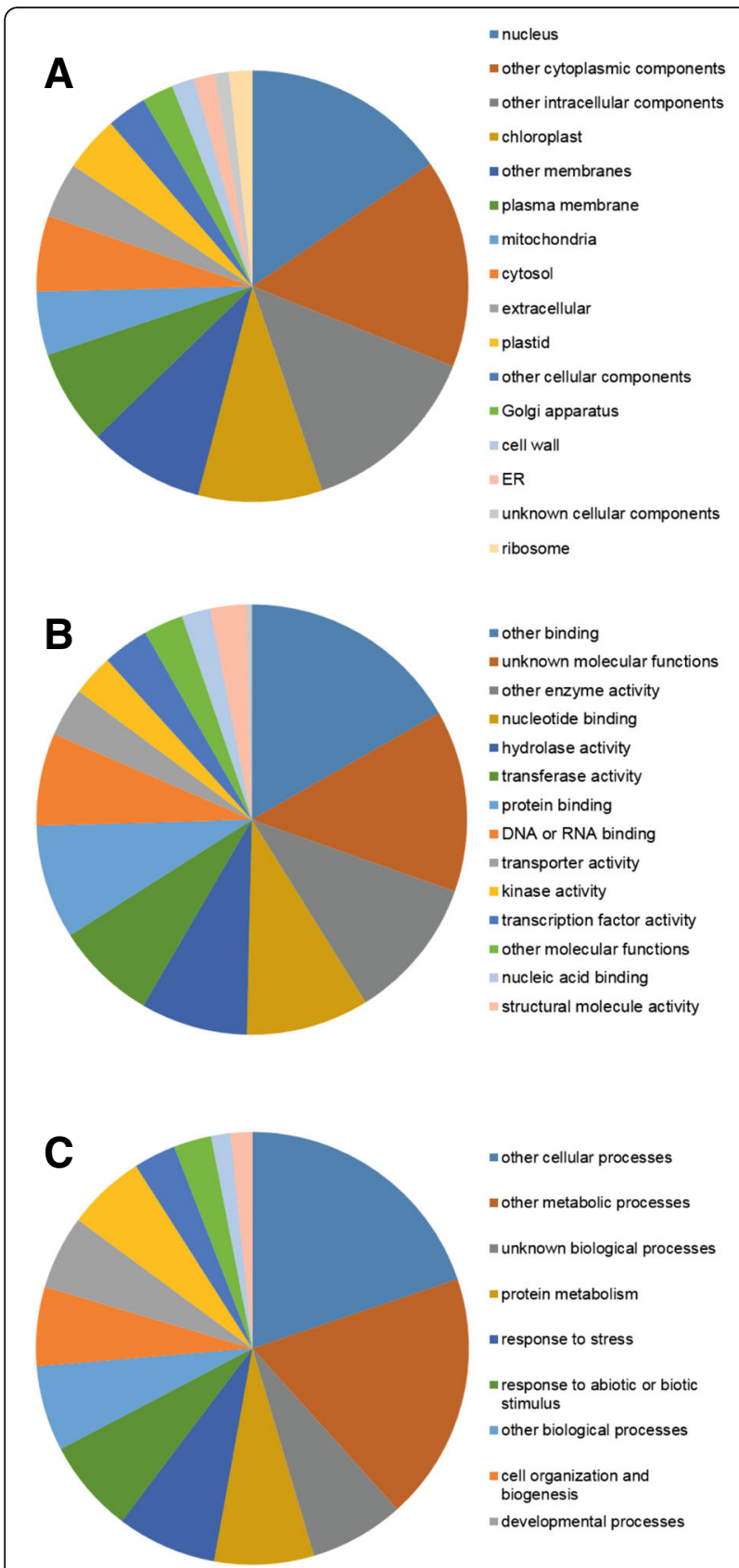

Fig. 2 Classification of transcripts into functional categories according to Arabidopsis Gene Ontology. a cellular component; b molecular function; and c biological process

proportion of genes assigned to GO terms was similar to that of the Arabidopsis gene annotation.

\section{The concept of gene "cluster"}

Because chrysanthemums are allohexaploid, it is generally supposed that three exceedingly similar genes form a type of a "cluster." In other words, the large number of contigs identified in this study could be consolidated into approximately one-third of the number of gene clusters. For instance, we found 12 putative orthologous contigs of 1-Deoxy-D-xylulose-5-phosphate synthase (DXS) genes in our chrysanthemum contig set, but they were classified into four groups or gene clusters according to their homologies (Fig. 3a). As shown in the alignments, it is natural to speculate that the contigs in each cluster belong to the same gene class derived from different or the same diploid set (Fig. 3a, b, and c). Thus, we attempted to classify ca. 131,500 contigs that had obvious homology to a particular Arabidopsis gene (Evalue of BLASTX <1.0e-5), depending on the mutual homology. We defined a gene cluster as a set of contigs which top-hit to the same Arabidopsis gene and had high homology (E-value of TBLASTN $<1.0 \mathrm{e}-100$ when the longest open translated reading frame was employed as a query) to at least one of the other contigs in the same cluster (Fig. 4). As a result, we defined 39,221 gene clusters, which were almost one-third (30\%) of the input and was comparable to the number of all genes in other plant species (Additional file 1: Table S1). This concept is useful in estimating the number of orthologous gene classes of known genes in chrysanthemums.

\section{Transcription factors}

TFs play important roles not only in the establishment of floral organ identity but also in the characterization of floral traits, such as colors, scent, and morphology. We had previously performed molecular breeding using TF genes to modify these floral traits in floricultural plants, such as torenia, rose, gentian, lisianthus, cyclamen, chrysanthemum, and morning glory [25, 26]. However, until now, the information of chrysanthemum TFs has been poorly characterized. In TFs, the sequences that encode DNA binding domains are relatively conserved among orthologs of other plant species, but the sequence similarity in other regions between different species is generally low. Therefore, cloning the full-length of TFs was difficult by the time of the emergence of the next-generation sequencing technology, and their functional analysis progressed slowly. In this study, we confirmed that almost all TF families that had been reported in Arabidopsis [13] were also conserved in chrysanthemums (Table 3). Our data sets contain 6996 TF contigs, which are consolidated into 2375 TF clusters (Table 3). For the classification of the TFs in chrysanthemums, we employed the information of Arabidopsis TFs in the PlantTFDB database [12].

To further examine whether TFs specifically expressed in floral organs were included in our chrysanthemum contig sets, we searched the contigs for class-B MADSbox genes, DEFICIENS (DEF)/APETALA3 (AP3), and GLOBOSA (GLO)/PISTILLATA (PI) that play important roles in petal development [27-31]. In chrysanthemums, two class-B genes, CDM115 (DEF/AP3) and CDM86 

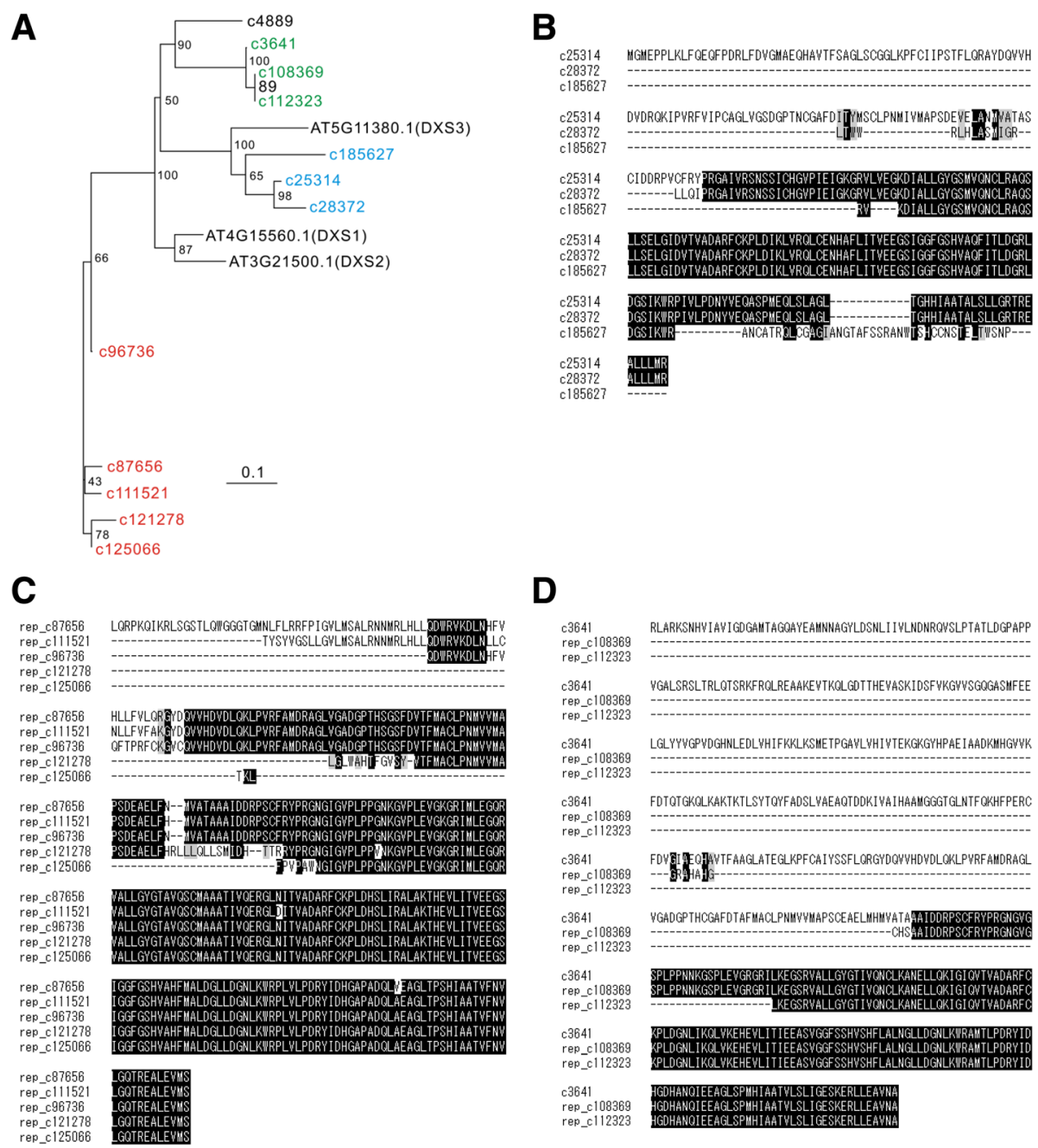

D

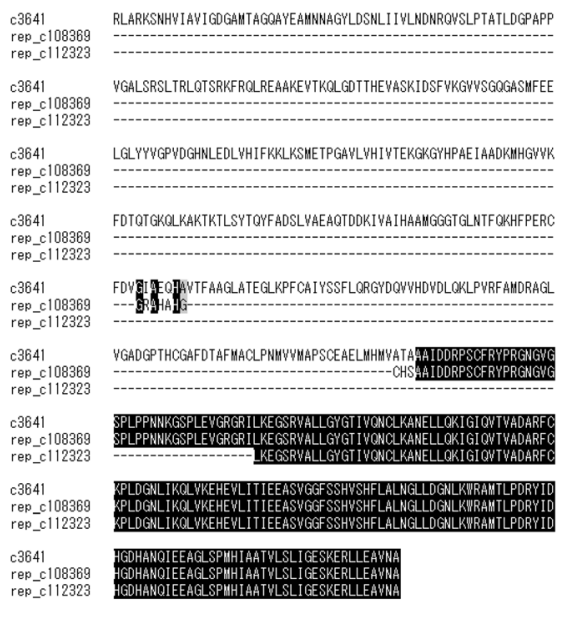

Fig. 3 Classification of DXS genes. a Phylogenetic tree of DXS genes of Arabidopsis and chrysanthemum. According to our definition, same-colored contigs are classified into the same cluster. b- $d$ Amino-acid alignment of the contigs in each cluster. Contigs in the same cluster share very high sequence homology

$(G L O / P I)$, have been previously registered in the NCBI GenBank, and these genes were also found in our contigs. In addition to CDM115 and CDM86, three class B MADS-box clusters were newly found in our contig sets. Among them, the TM6 gene [32] of the DEF/AP3 subgroup was identified for the first time in a chrysanthemum. A phylogenetic relationship was examined using class-B MADS-box proteins derived from chrysanthemums and other plant species (Fig. 5). In the phylogenetic analysis, the most homologous contig to Arabidopsis ortholog was used as a representative of the clusters classified in this study. The analysis results suggested that EST data in this study contained all types of class-B subgroups. Chrysanthemum DEF/AP3 was classified into the euAP3 group and possessed C-terminal euAP3 motifs, which are unique for dicots [33]. In petunia, PhDEF and PhTM6 proteins exhibit functional divergence [32]; hence the chrysanthemum TM6 and DEF/AP3 proteins may also have different roles in floral organ development. In chrysanthemums, the function of MADS-box proteins in floral organ-morphogenesis has been poorly understood. Our EST data would contribute to the future research in this area. In addition, our report reveals the causal genes and/or important factors that are involved in formation of floral traits, such as petal shape, floral architecture, and color patterns in various floral types of chrysanthemum cultivars regarding the TF functions.

\section{Carotenoid metabolism}

Carotenoids are widely distributed $\mathrm{C} 40$ isoprenoid pigments. They play an important role in photosynthesis and provide flowers and fruits with yellow, orange, and red colors. Yellow ray petals of chrysanthemums 


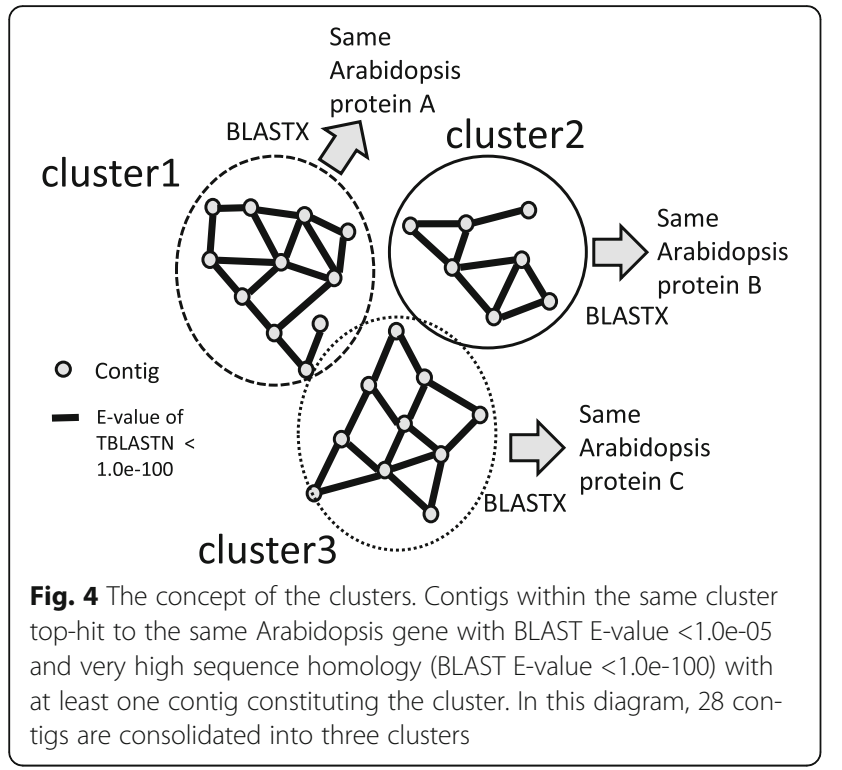

accumulate substantial amounts of carotenoids, mainly luteins and lutein epoxides [20]. Contigs of all the steps of carotenoid biosynthesis are presented in our chrysanthemum EST data (Fig. 6). Kishimoto et al. [34] reported sequences of full-length cDNAs encoding carotenoid biosynthesis enzymes in chrysanthemums, including phytoene synthase (PSY), phytoene desaturase (PDS), $\zeta$ carotene desaturase (ZDS), carotenoid isomerase (CRTISO), lycopene $\beta$-cyclase (LCYB), lycopene $\varepsilon$ cyclase (LCYE), $\beta$-ring hydroxylase (CHYB), violaxanthin deepoxidase (VDE), and zeaxanthin epoxidase (ZEP). In addition to contig-encoding these enzymes, we newly identified contigs for 15-cis-ל-CRTISO (Z-ISO), neoxanthin synthase (NSY), and P450-type hydroxylases, CHYB/CYP97A, and $\varepsilon$-ring hydroxylase (CHYE/ CYP97C). The EST data contained 2-10 contigs for each enzyme (Fig. 6). Importantly, the sequence comparison revealed that the contigs encoding ZDS, CRTISO, ZISO, CHYE/CYP97A, CHYB/CYP97C, VDE, and NSY showed more than $90 \%$ identities within each enzyme. Other enzymes, including PSY, PDS, LCYB, LCYE, CHYB, and ZEP had contigs that showed less than 70\% identity to the previously reported full-length cDNA sequences [34]. Carotenoid biosynthesis is highly regulated in a tissue-specific manner. The different patterns of carotenogenic gene expression between leaves and petals are partly due to the existence of multiple homologs of rate-limiting enzymes. For example, multiple homologs of PSY, $L C Y B$, and $C H Y B$ are present in tomato and are expressed in a tissue-specific manner [35]. It is of great interest to elucidate the tissue specificity of the multiple types of ESTs for the carotenoid biosynthesis enzymes found in the chrysanthemum EST data.
Table 3 Classification of TF families in chrysanthemum

\begin{tabular}{|c|c|c|c|c|}
\hline & TF Family & Contig & Cluster & Arabidopsis \\
\hline 1 & AP2 & 43 & 18 & 18 \\
\hline 2 & ARF & 129 & 57 & 22 \\
\hline 3 & ARR-B & 22 & 13 & 14 \\
\hline 4 & B3 & 111 & 53 & 66 \\
\hline 5 & BBR-BPC & 42 & 11 & 7 \\
\hline 6 & BES1 & 38 & 12 & 8 \\
\hline 7 & bHLH & 600 & 179 & 153 \\
\hline 8 & bZIP & 306 & 92 & 74 \\
\hline 9 & $\mathrm{C} 2 \mathrm{H} 2$ & 352 & 130 & 100 \\
\hline 10 & $\mathrm{C} 3 \mathrm{H}$ & 216 & 89 & 50 \\
\hline 11 & CAMTA & 52 & 18 & 6 \\
\hline 12 & co-like & 80 & 27 & 17 \\
\hline 13 & CPP & 14 & 5 & 8 \\
\hline 14 & DBB & 68 & 13 & 11 \\
\hline 15 & Dof & 112 & 42 & 36 \\
\hline 16 & E2F/DP & 17 & 13 & 8 \\
\hline 17 & EIL & 28 & 10 & 6 \\
\hline 18 & ERF & 644 & 187 & 123 \\
\hline 19 & FAR1 & 76 & 57 & 17 \\
\hline 20 & G2-like & 124 & 50 & 42 \\
\hline 21 & GATA & 130 & 40 & 30 \\
\hline 22 & GeBP & 49 & 18 & 22 \\
\hline 23 & GRAS & 152 & 71 & 34 \\
\hline 24 & GRF & 14 & 9 & 9 \\
\hline 25 & HB-other & 27 & 16 & 7 \\
\hline 26 & HB-PHD & 5 & 4 & 2 \\
\hline 27 & HD-ZIP & 223 & 71 & 48 \\
\hline 28 & HRT-like & 1 & 1 & 2 \\
\hline 29 & HSF & 119 & 38 & 24 \\
\hline 30 & LBD & 83 & 37 & 43 \\
\hline 31 & LFY & 1 & 1 & 1 \\
\hline 32 & LSD & 52 & 7 & 3 \\
\hline 33 & MIKC_MADS & 496 & 62 & 42 \\
\hline 34 & M-type_MADS & 19 & 9 & 66 \\
\hline 35 & MYB & 509 & 198 & 144 \\
\hline 36 & MYB_related & 247 & 86 & 66 \\
\hline 37 & NAC & 338 & 118 & 113 \\
\hline 38 & $N F-X 1$ & 12 & 5 & 2 \\
\hline 39 & NF-YA & 30 & 11 & 10 \\
\hline 40 & $N F-Y B$ & 110 & 19 & 13 \\
\hline 41 & NF-YC & 52 & 14 & 14 \\
\hline 42 & Nin-like & 221 & 113 & 14 \\
\hline 43 & NZZ/SPL & 0 & 0 & 1 \\
\hline 44 & RAV & 7 & 4 & 6 \\
\hline
\end{tabular}


Table 3 Classification of TF families in chrysanthemum (Continued)

\begin{tabular}{lllll}
\hline 45 & S1Fa-like & 0 & 0 & 3 \\
46 & SAP & 0 & 0 & 1 \\
47 & SBP & 115 & 44 & 17 \\
48 & SRS & 23 & 9 & 11 \\
49 & STAT & 2 & 2 & 2 \\
50 & TALE & 126 & 38 & 21 \\
51 & TCP & 76 & 37 & 24 \\
52 & Trihelix & 165 & 48 & 29 \\
53 & VOZ & 12 & 4 & 2 \\
54 & Whirly & 9 & 2 & 3 \\
55 & WOX & 20 & 9 & 16 \\
56 & WRKY & 340 & 124 & 72 \\
57 & YABBY & 69 & 8 & 6 \\
58 & zf-HD & 68 & 22 & 17 \\
& & 6996 & 2375 & 1726 \\
\hline
\end{tabular}

Specific enzymatic cleavage of carotenoids produces various types of apocarotenoids, some of which have important biological functions in the growth and development of plants [36]. In Arabidopsis, enzymes that cleave carotenoids fall into nine clades [37]. Five of these, the 9-cis epoxycarotenoid dioxygenases (NCED2, NCED3, NCED5, NCED6, and NCED9), are involved in the biosynthesis of the plant hormone abscisic acid. The remaining four, carotenoid cleavage dioxygenases (CCD1, CCD4, CCD7, and CCD8), have low sequence homologies to the NCEDs, and their enzyme activities and substrate specificities also differ from those of the NCEDs [36]. Orthologs that belong to the NCED3, CCD4, and CCD1 subfamilies have previously been identified in chrysanthemums [38, 39]. In this examination, apart from the ESTs encoding these enzymes, we newly identified contigs for NCED5 and NCED6 in the chrysanthemum EST data. We found only a single short CCD7 EST, but could not identify CCD8 EST. CCD7 and CCD8 are involved in the synthesis of the plant hormone strigolactone, which is mainly synthesized in roots [40, 41]. CCD7 and CCD8 transcripts were not contained in the EST database, possibly because the EST database was constructed using RNAs obtained from aerial organs.

In chrysanthemums, $C C D 4 a$ is expressed specifically in ray petals, and its translated product cleaves carotenoids into colorless compounds, thus resulting in a white petal color [38]. Chrysanthemums have another type of CCD4 (designated $C C D 4 b$ ), which is expressed mainly in leaves and stems, although its function is unknown [38]. We found nine $C C D 4 a$ and seven $C C D 4 b$ contigs. In addition, we newly found seven $C C D 4$ contigs whose sequences showed low identities $(<62 \%)$ to both $C C D 4 a$ and $C C D 4 b$ (designated $C C D 4 c$; Fig. 7). We also performed phylogenetic analyses using amino-acid sequences that were translated by the longest ORF with high homology to a particular Arabidopsis gene (Fig. 7; selection criteria are shown in Methods section). In the chrysanthemum cultivar 'Jimba', at least $6 C C D 4 a$ homologs (CmCCD4a-1-6) were identified [39]. In this study, we identified CCD4a-1, -2 , and -5 homologs in our contig set that was made from cultivar 'Sei-Marine' (Fig. 7). In addition, a short CCD4a-3 contig (DJ0055_rep_c164774) was also detected in the contig set, although it was not used for phylogenetic analysis. These results are consistent with the findings of a previous study that revealed that the number of CCD $4 a$ homologs expressed in petals differs among chrysanthemum cultivars [42].

\section{Terpene biosynthesis}

The main compounds that characterize the floral scent of chrysanthemums are volatile isoprenoids, such as monoterpenes (C10) and oxygenated monoterpenes [22]. We established that sesquiterpenes (C15) are also present as minor compounds. These low-molecularweight terpenes are enzymatically synthesized from the 5-carbon precursors isopentenyl diphosphate and its isomer, dimethyl diphosphate, both of which are derived from the methylerythritol phosphate (MEP) pathway or the mevalonate (MEV) pathway (Fig. 8) [43]. We investigated genes that encoded enzymes that belonged to the terpene-biosynthesis pathway in our chrysanthemum EST database and found that all the genes were included in the database (Fig. 8). The EST data contained 2-46 contigs for each enzyme. In higher plants, the putative numbers of terpene synthase (TPS) genes per genome range from approximately 20 to 150 [44]. In the chrysanthemum EST, we identified 46 TPS contigs, which were consolidated into 30 clusters. Thus, these numbers of clusters were fell within the range (20 to 150) (Fig. 8) [44]. Many chrysanthemum contigs that encoded MEP and MEV pathway enzymes were highly homologous to those of Artemisia annua that belonged to Asteraceae.

The TPS family is responsible for the synthesis of various monoterpene compounds. Figure 9 represents the phylogenetic tree based on amino-acid sequence comparisons of TPS between chrysanthemums and other plant species. In the phylogenetic analysis, the most homologous contig to the Arabidopsis ortholog was used as a representative of the clusters classified in this study. Of the 30 clusters defined, 29 clusters were used in the phylogenetic analysis because one cluster (contig) had only short amino-acid sequences (the amino-acid length was $<20 \%$ of the alignment coverage). Currently, seven major TPS subfamilies, designated TPS-a through TPS- 


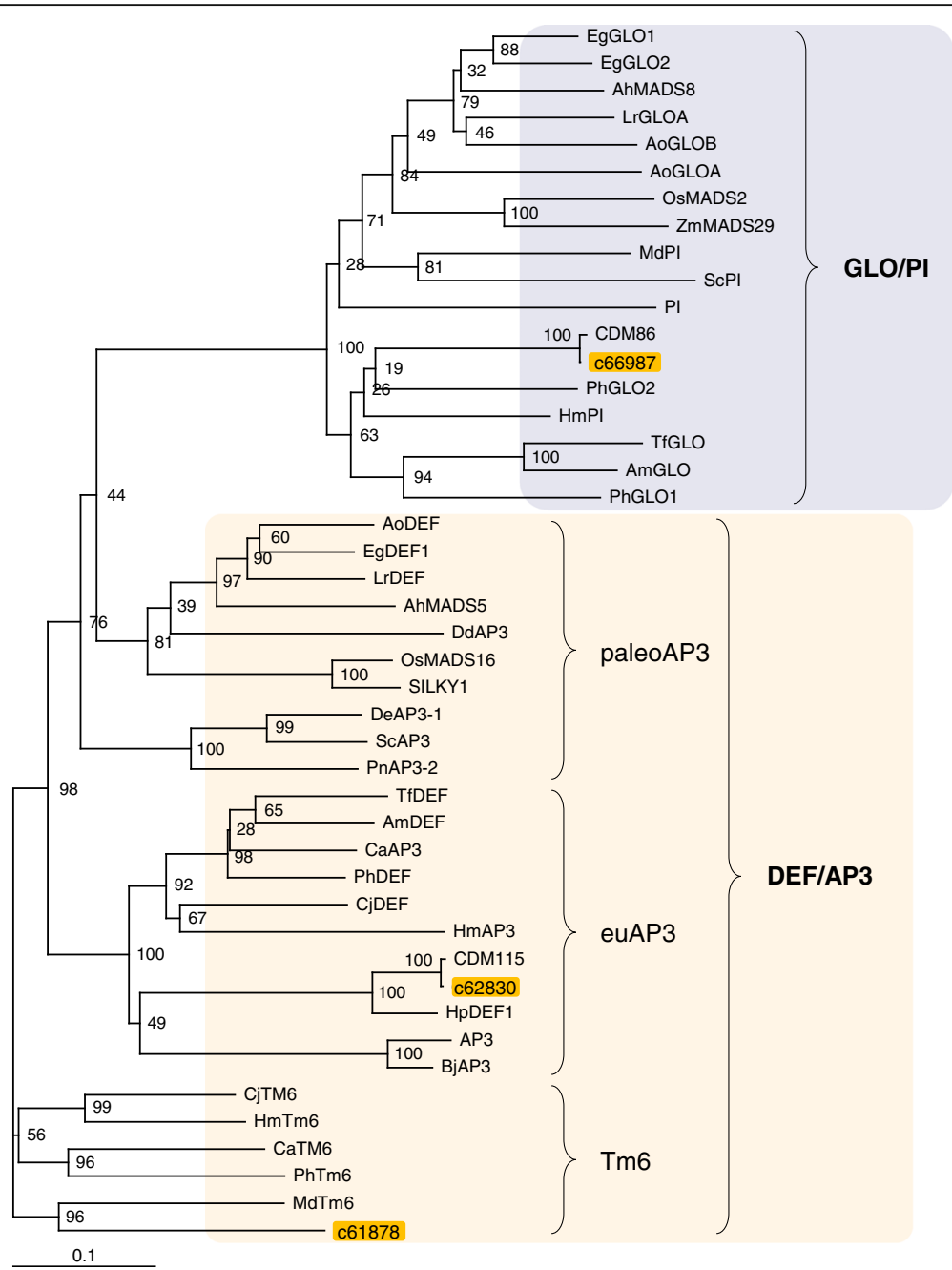

Fig. 5 Phylogenetic tree of chrysanthemum class-B MADS-box proteins. Deduced amino-acid sequences of class-B proteins found in the chrysanthemum EST data (Cluster ID of our data) and those in other plant species (Additional file 2: Table S2) were compared and a phylogenetic tree was constructed using the neighbor-joining method. For the phylogenetic analysis, a chrysanthemum contig that was the most homologous to the Arabidopsis ortholog at the amino-acid levels was used as a representative of the clusters in this study

g, are recognized in plants [44-48]. Most of the chrysanthemum TPS appeared to fall into the same clades as TPS-a or -b subfamilies, except for one EST that was classified into the TPS-g subfamily. This result was consistent with the previously reported data that TPS-a, -b, and -g subgroups are specific to angiosperms.

TPSs have an ability to synthesize multiple terpenes from a single substrate. The chemical diversity of the terpenes produced in chrysanthemum flowers may be mainly attributed to such unique enzyme activity of TPS. Future studies should focus on the identification and functional characterization of the TPS genes involved in the floral scent formation in chrysanthemums.

\section{Conclusion}

In this study, we performed an EST sequencing analysis in a chrysanthemum and obtained 213,204 assembled contigs. These contigs were further consolidated into 39,221 clusters, which were comparable to the number of all genes in other plant species. In addition, the contigs in this study contained a comparable number of TFs and genes involved in petal colors and aroma compound biosynthesis. These results suggest that our EST data set is a valuable reference for the molecular analysis of chrysanthemums. Chrysanthemums have a large and allohexaploid genome, which hinders genomic sequencing. However, the recent progress in sequencing technology may facilitate the genomic sequencing of chrysanthemums in the near future. However, genomic structures and/or sequences are different among chrysanthemum cultivars due to their self-incompatibility, and each cultivar is not a pure line. Therefore, continuous efforts by using EST and/or genomic sequencing analyses of multiple chrysanthemum cultivars are necessary for 


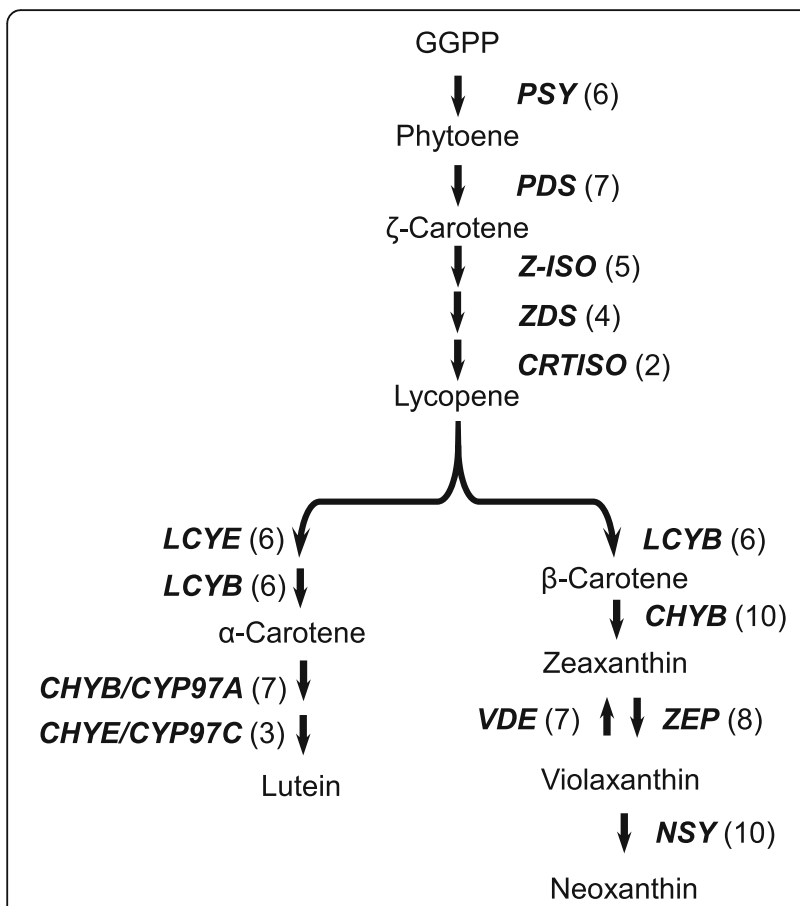

Fig. 6 Distribution of chrysanthemum ESTs in the carotenoid biosynthesis pathway. Each enzyme name is followed, in parentheses, by the number of contigs homologous to gene families that encode this enzyme. PSY, phytoene synthase; PDS, phytoene desaturase; Z-ISO, 15-cis-Z-CRTISO; ZDS, Z-carotene desaturase; CRTISO, carotenoid isomerase; LCYB, lycopene $\beta$-cyclase; LCYE, lycopene $\varepsilon$-cyclase; CHYB, $\beta$-ring hydroxylase; $C H Y E$, $\varepsilon$-ring hydroxylase; ZEP, zeaxanthin epoxidase; VDE, violaxanthin de-epoxidase; and NSY, neoxanthin synthase

further understanding of the chrysanthemum genome. We believe that our EST analysis that employs the concept of gene clusters could help to elucidate not only the specifics of chrysanthemum genes but also those of other higher polyploid plants.

\section{Methods}

\section{Plant materials and RNA extraction}

A Chrysanthemum morifolium cultivar 'Sei-Marine' (provided by Inochio Seikoen Co., Ltd.; http://www.seikoenkiku.co.jp/) was used in this study. The chrysanthemum was grown in a greenhouse under natural daylight conditions. We used the following plant organs as materials for sequencing analysis: mature corolla of ray floret and immature corolla of ray floret (including pistils) obtained from 5 to $8 \mathrm{~mm}$ flower buds, mature corolla of disk floret and immature corolla of disk floret (including pistils and androecium) obtained from 5 to $8-\mathrm{mm}$ flower buds, shoot apices, flower buds under $4 \mathrm{~mm}$, stems, leaves, phyllary, blossom end, pistils of ray florets and disk florets, and androecia of disk florets. These materials were prepared from several independent chrysanthemum plant lines and/or flowers. Total RNAs from these organs were extracted using TRIzol (Thermo Fisher Scientific; https:// www.thermofisher.com/jp/ja/home.html). The total RNAs were further purified by an RNeasy mini-kit (QIAGEN; https://www.qiagen.com/jp/). RNA concentration was estimated with an ND-1000 spectrophotometer (NanoDrop) (Thermo Scientific; Wilmington, DE, USA).

\section{Preparations of cDNA libraries for $\mathbf{4 5 4}$ sequencing}

For 454 sequencing, a normalized cDNA library was prepared. Total RNAs isolated from each chrysanthemum organ were combined in an attempt to maximize the diversity of the transcriptional units and were used as a material for cDNA synthesis. We used the following four independent cDNA libraries (Table 1).

For the first cDNA library, the cDNA was prepared using total RNAs derived from floral organs. The cDNA synthesis and normalization were performed by the following procedure. The first strand cDNA synthesis of the first library was synthesized with the following oligonucleotide: 5' -CAAGCAGAAGACGGCATACGAC TGGAGTTTTTTTTTTTTTTTTVN-3'. Next, the diol group of the synthesized cDNA/RNA hybrid was oxidized, followed by biotinylation. After digestion with RNase I, the cDNA/RNA hybrid was purified using the cap-trapping method, and full-length single-strand cDNAs were obtained. A 5 ' adaptor (Additional file 6: Table S6) was attached to the cap-trapping single-strand cDNAs, and second-strand cDNAs were synthesized using the following oligonucleotide: 5' -AATGATACGGCGCTGGAGGACAGGTTCAGAGTTC-3'. Further, the prepared double-strand CDNA was heat-denatured; the re-hybridized double-strand cDNAs indicated highly accumulated transcripts in the library that were digested with double-strandspecific DNA nuclease. The single-strand cDNAs, which were obtained by the above manipulation, were collected as normalized cDNAs. Next, second-strand cDNAs were synthesized using the normalized single-strand cDNAs.

The cDNAs for the second, third, and fourth cDNA libraries were synthesized using total RNAs derived from the aerial part of the chrysanthemum with a Mint-2 cDNA synthesis kit (evrogen; http://evrogen.com/) according to the manufacturer's instructions. The first strand cDNAs were synthesized utilizing the following oligonucleotide: 5 '-attctagaggccgaggcggccgacatgTTTTT TTTTTTTTTTV-3'. After the second-strand cDNA synthesis, the cDNA libraries were amplified using a primer set consisting of $3^{\prime}$ PCR primer $2\left(5^{\prime}\right.$-ATTCTA GAGGCCGAGGcggccgac-3') and 5' PCR primer (5'aagcagtggtatcaacgcagagt- $\left.3^{\prime}\right)$ under the following conditions: $95^{\circ} \mathrm{C}$ for $2 \mathrm{~min}$, followed by 18 cycles of $95^{\circ} \mathrm{C}$ for $15 \mathrm{~s}, 66{ }^{\circ} \mathrm{C}$ for $20 \mathrm{~min}$, and $72{ }^{\circ} \mathrm{C}$ for $6 \mathrm{~min}$. The amplified cDNA libraries were normalized by the TRIMMERDIRECT cDNA Normalization Kit (evrogen) according to the manufacturer's guidelines. The normalized cDNA libraries were further amplified using the primer sets 


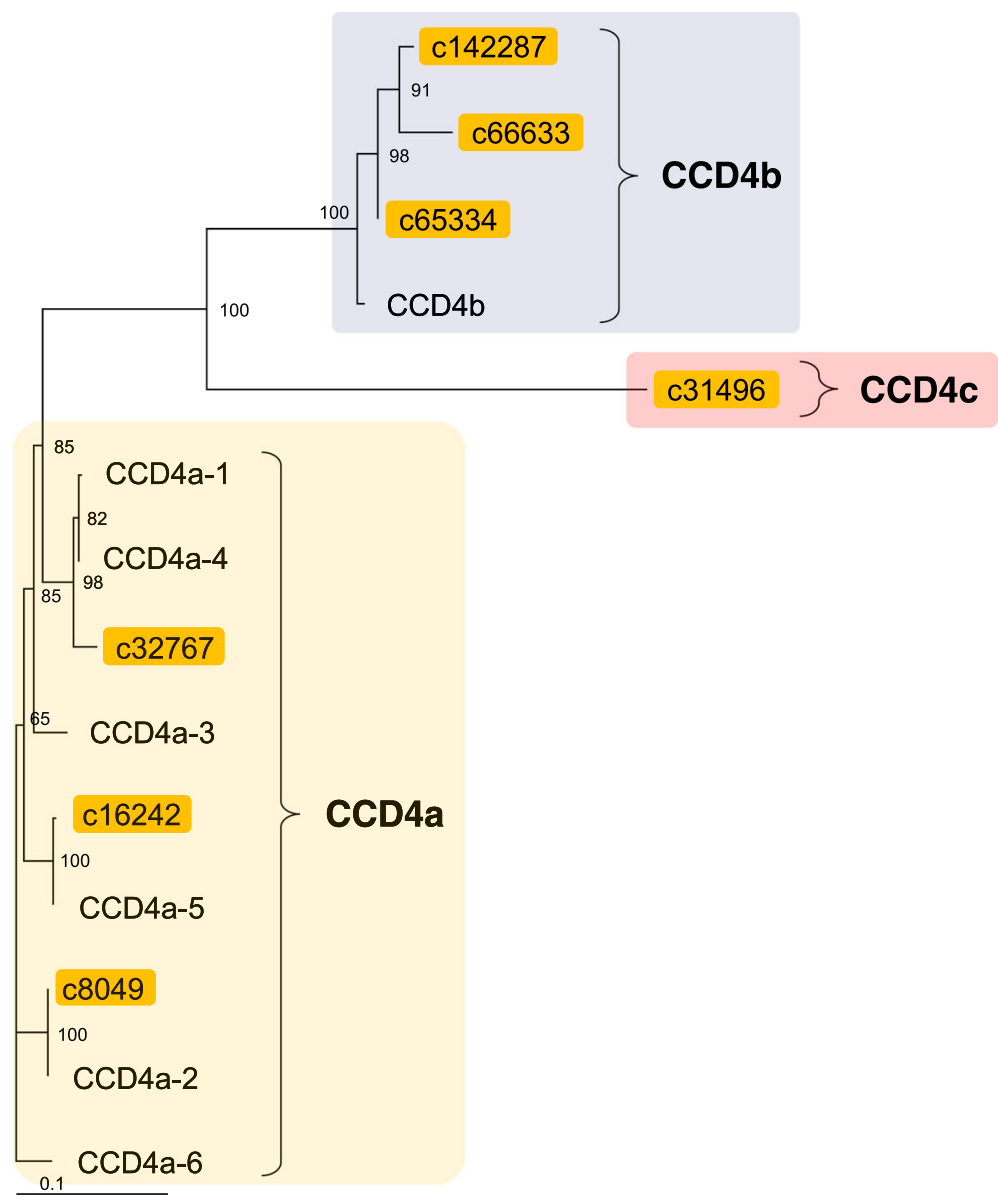

Fig. 7 A phylogenetic tree of the chrysanthemum CCD4. Deduced amino-acid sequences of CCD4 found in the chrysanthemum EST database (indicated by EST IDs) and those previously identified in the chrysanthemum cultivar 'Jimba' (indicated by GenBank accession numbers in Additional file 4: Table S4) were compared, and a phylogenetic tree was constructed using the neighbor-joining method. 4a, 4b, and 4c indicates CCD4a, CCD4b, and CCD4c subfamilies, respectively

respective to each type of the cDNA libraries. We prepared three types of normalized cDNA libraries derived from the aerial part of chrysanthemum: (1) full-length cDNA libraries (second), (2) 3' cDNA library (third), and (3) 5' cDNA library (fourth). The full-length second-cDNA library was amplified using the $3^{\prime}$ PCR primer 2 and the $5^{\prime}$ PCR primer under the following conditions: $95^{\circ} \mathrm{C}$ for $2 \mathrm{~min}$, followed by 16 cycles of $95^{\circ}$ $\mathrm{C}$ for $15 \mathrm{~s}, 66^{\circ} \mathrm{C}$ for $20 \mathrm{~min}$, and $72{ }^{\circ} \mathrm{C}$ for $6 \mathrm{~min}$. The third- and fourth cDNA libraries were also amplified using the 3' PCR primer 2 and the 5' PCR primer under the following conditions: $95{ }^{\circ} \mathrm{C}$ for $2 \mathrm{~min}$, followed by 17 cycles of $95{ }^{\circ} \mathrm{C}$ for $15 \mathrm{~s}, 66{ }^{\circ} \mathrm{C}$ for $20 \mathrm{~min}$, and $72{ }^{\circ} \mathrm{C}$ for 6 min. A biotin-labeled 3' PCR primer 2 was used for the amplification of the 3' cDNA library. A biotinlabeled 5' PCR primer was used for the amplification of the 5' cDNA library. These biotin-labeled cDNA libraries were purified through Dynabeads M-280 Streptavidin (Thermo Fisher Scientific) according to the manufacturer's instructions. The biotin-labeled cDNA libraries were purified and recovered as single-strand cDNAs, and double-strand DNAs were synthesized by KOD-Plus-Ver.2 (TOYOBO; http://www.toyobo-global.$\mathrm{com} /$ ). The isolated double-strand DNAs were fragmented to an approximate length of $800 \mathrm{bp}$ and subjected to sequencing analysis by the LE220 Focused-ultrasonicator (Covaris; http://covarisinc.com/).

\section{Sequencing and data analysis}

Sequencing was performed by the 454 FLX titanium system (first library; Table 1) and the GS FLX+ system (second, third, and fourth libraries; Table 1) (Roche; https://roche-biochem.jp/index.html) according to the manufacturer's instructions. The 454 sequences were trimmed of adaptor and low-quality sequences. The sequences were then assembled and annotated by BLASTN searches of the NCBI database. 


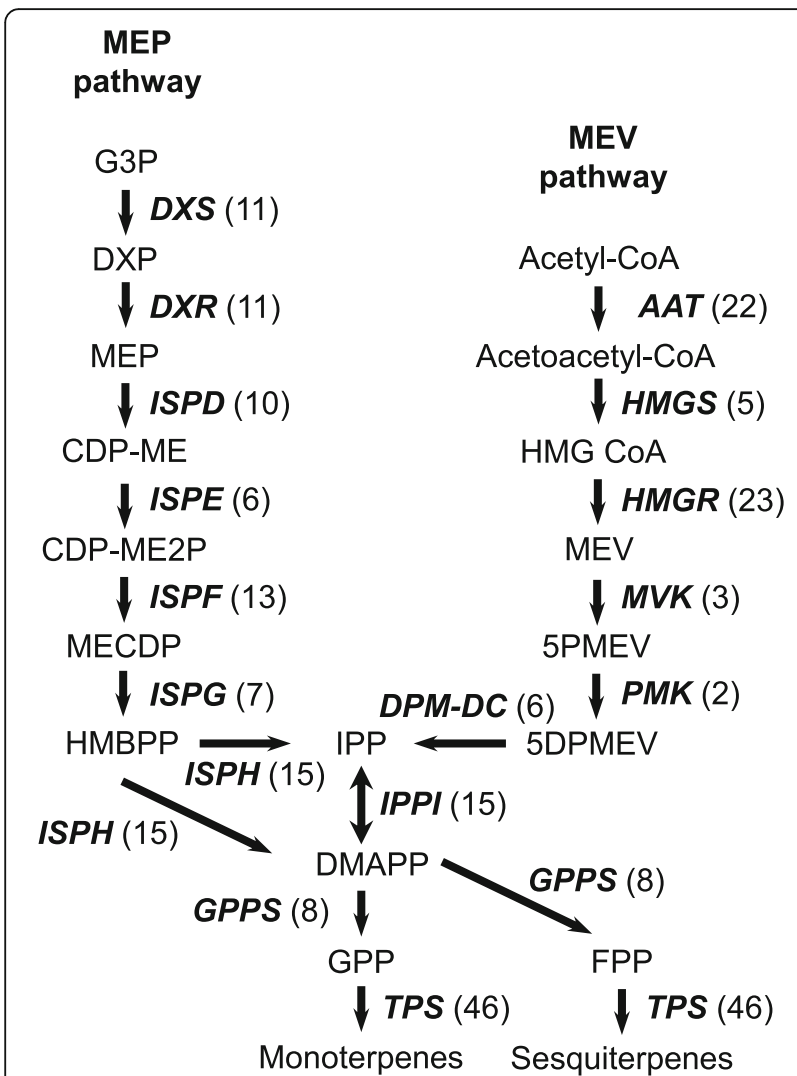

Fig. 8 Distribution of chrysanthemum ESTs in the terpenoid biosynthetic pathway. Each enzyme name is followed, in parentheses, by the number of contigs homologous to the gene families that encode this enzyme. MEP, 2-C-Methyl-D-erythritol 4-phosphate; MEV, Mevalonate; G3P, Glyceraldehyde 3-phosphate; DXP, 1-Deoxy-D-xylulose 5-phosphate; CDP-ME, 4-(Cytidine 5'-diphospho)-2-C-methyl-D-erythritol; MECDP, 2-CMethyl-D-erythritol-2,4-cyclodiphosphate; HMBPP, (E)-4-Hydroxy-3methylbut-2-en-1-yl diphosphate; IPP, Isopentenyl diphosphate; DMAPP, Dimethylallyl diphosphate; GPP, Geranyl diphosphate; FPP, Farnesyl diphosphate; HMG-COA, (S)-3-Hydroxy-3-methylglutaryl-COA; DXS, 1Deoxy-D-xylulose-5-phosphate synthase; DXR, 1-Deoxy-D-xylulose-5phosphate reductoisomerase; ISPD, 2-C-Methyl-D-erythritol 4-phosphate cytidylyltransferase; ISPE, 4-(Cytidine 5'-diphospho)-2-C-methyl-D-erythritol kinase; ISPF, 2-C-Methyl-D-erythritol 2,4-cyclodiphosphate synthase; ISPG, 4-Hydroxy-3-methylbut-2-en-1-yl diphosphate synthase; ISPH, 4hydroxy-3-methylbut-2-enyl diphosphate reductase; IPPI, Isopentenyl diphosphate isomerase; AAT, Acetyl-CoA acetyltransferase; HMGS, HMG-CoA synthase; HMGR, HMG-CoA reductase; MVK, Mevalonate kinase; PMK, Phosphomevalonate kinase; DPM-DC, Diphosphomevalonate decarboxylase; GPPS, GPP synthase or FPP synthase; and TPS, Terpene synthase

\section{Functional annotation}

To annotate the contig sequences, each contig was searched against the Arabidopsis protein database (TAIR10) from The Arabidopsis Information Resources (TAIR; http://www.arabidopsis.org) using the BLASTX program with an E-value cut-off of 1e-5. Annotation of the best-hit Arabidopsis protein was used as an annotation of each contig. Functional classification of the contigs was performed based on the Gene Ontology (GO) terms from the Arabidopsis GO Slim classification in TAIR.

\section{Phylogenetic analyses and selection of chrysanthemum orthologs}

For the phylogenetic analyses, protein sequences were aligned using MAFFT (http://mafft.cbrc.jp/alignment/software/; Accessed Aug 23, 2017). After these sequences had been aligned, they were used for phylogenetic analysis by the neighbor-joining method in Clustal W. Statistical significance was tested by bootstrap analysis for 1000 replicates. Amino-acid sequences of other plant species were obtained from NCBI (http://www.ncbi.nlm.nih.gov/nuccore; Accessed Aug 23, 2017, see Additional files 1, 3, 4, and 5 for accession numbers: Table S1, S2, S3, and S5). The phylogenetic analyses were performed using the chrysanthemum amino-acid sequences, which were translated using the longest ORF with high homology to a particular Arabidopsis gene (threshold E-value of BLASTX <1.0e-5, threshold score $>150$, and minimum alignment coverage of $20 \%$ of the query sequences).

Chrysanthemum ortholog genes in Figs. 6 and 8 were also selected using a threshold E-value of BLASTX $<1.0 \mathrm{e}-5$, a threshold score $>150$, and minimum alignment coverage of $20 \%$ of the query sequences.

\section{Accession numbers}

Raw sequencing data of chrysanthemum with the 454 sequencing were submitted to the DDBJ Sequence Read Archive (DRA; http://trace.ddbj.nig.ac.jp/dra/index.html; Accessed Aug 23, 2017) with the accession numbers (DRR088291DRR088294). Assembled transcripts derived from the raw sequencing data were further submitted to the Mass Submission System of DDBJ (MSS; http://www.ddbj.nig.ac.jp/sub/ mss_flow-j.html; Accessed Aug 23, 2017) with accession numbers IABW01000001-IABW01213204 (213,204 entries).

\section{Additional files}

Additional file 1: Table S1. Annotations of contigs and clusters in this study. (XLSX $18507 \mathrm{~kb}$ )

Additional file 2: Table S2. Gene accessions used in a phylogenetic tree of class-B proteins. (XLSX 26 kb)

Additional file 3: Table S3. Gene accessions used in the phylogenetic tree of CCD4 proteins. (XLSX $12 \mathrm{~kb}$ )

Additional file 4: Table S4. Codes for searching the homologous contigs in chrysanthemum. (XLSX 14 kb)

Additional file 5: Table S5. Gene accessions used in the phylogenetic tree of TPS proteins. (XLSX $28 \mathrm{~kb}$ )

Additional file 6: Table S6. Sequences of an adaptor. (XLSX 8 kb)

\section{Abbreviations}

AAT: Acetyl-CoA acetyltransferase; AP3: APETALA3; CCD: Carotenoid cleavage dioxygenases; CDP-ME: 4-(Cytidine 5'-diphospho)-2-C-methyl-D-erythritol; CHYB: $\beta$ ring hydroxylase; $\mathrm{CHYE}$ : $\varepsilon$-ring hydroxylase; CRTISO: Carotenoid isomerase; DEF: DEFICIENS; DMAPP: Dimethylallyl diphosphate; DPM- 


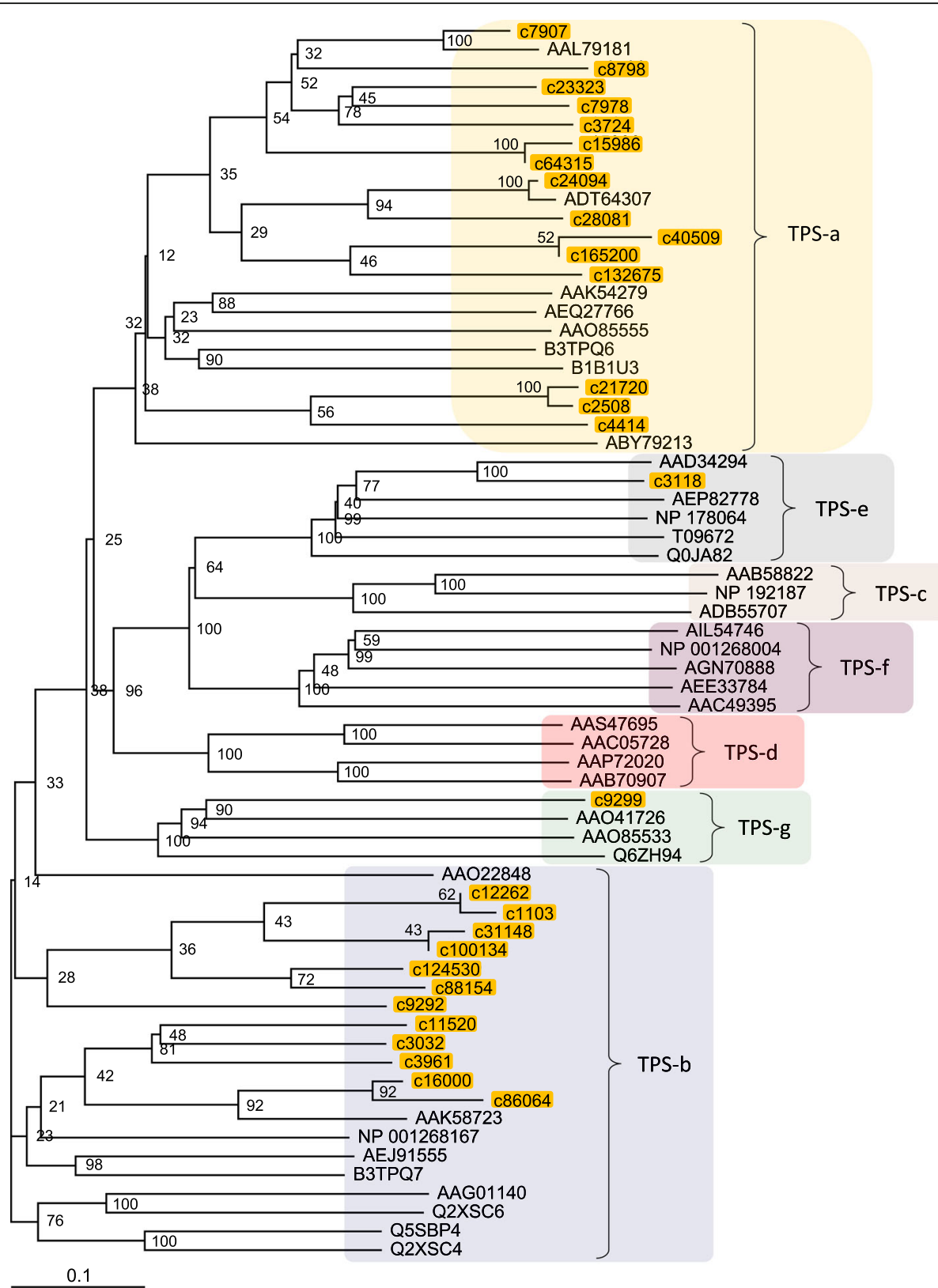

Fig. 9 A phylogenetic tree of terpene synthase (TPS). Deduced amino-acid sequences of chrysanthemum (Cluster ID of our data) and those of similar protein family members (Genbank accession number in Additional file 5: Table S5) previously identified in higher plants were compared, and a phylogenetic tree was constructed using the neighbor-joining method. For the phylogenetic analysis, the chrysanthemum contig that was the most homologous to the Arabidopsis ortholog at the amino-acid level was used as a representative of the clusters in this study

DC: Diphosphomevalonate decarboxylase; DXP: 1-Deoxy-D-xylulose 5-phosphate; DXR: 1-Deoxy-D-xylulose-5-phosphate reductoisomerase; DXS: 1-Deoxy-Dxylulose-5-phosphate synthase; EST: Expressed sequence tag; FPP: Farnesyl diphosphate; G3P: Glyceraldehyde 3-phosphate; GLO: GLOBOSA; GO: Gene ontology; GPP: Geranyl diphosphate; GPPS: GPP synthase; HMBPP: (E)-4-Hydroxy-3methylbut-2-en-1-yl diphosphate; HMG-CoA: (S)-3-Hydroxy-3-methylglutaryl-CoA; HMGR: HMG-CoA reductase; HMGS: HMG-CoA synthase; IPP: Isopentenyl diphosphate; IPPI: Isopentenyl diphosphate isomerase; ISPD: 2-C-Methyl-Derythritol 4-phosphate cytidylyltransferase; ISPE: 4-(Cytidine 5'-diphospho)-2-Cmethyl-D-erythritol kinase; ISPF: 2-C-Methyl-D-erythritol 2,4-cyclodiphosphate synthase; ISPG: 4-Hydroxy-3-methylbut-2-en-1-yl diphosphate synthase; ISPH: 4hydroxy-3-methylbut-2-enyl diphosphate reductase; LCYB: Lycopene $\beta$-cyclase; LCYE: Lycopene $\varepsilon$-cyclase; MECDP: 2-C-Methyl-D-erythritol-2,4-cyclodiphosphate; MEP: Methylerythritol phosphate; MEV: Mevalonate; MVK: Mevalonate kinase; NCED: 9-cis epoxycarotenoid dioxygenases; NSY: Neoxanthin synthase; PDS: Phytoene desaturase; PI: PISTILLATA; PMK: Phosphomevalonate kinase; PSY: Phytoene synthase; SNP: Single-nucleotide polymorphism; TF: Transcription factor; TPS: Terpene synthase; VDE: Violaxanthin deepoxidase; ZDS: Z-carotene desaturase; ZEP: Zeaxanthin epoxidase; ZISO: 15-cis-Z-CRTISO 


\section{Acknowledgments}

We are grateful to Inochio Seikoen Co., Ltd. (http://www.seikoen-kiku.co.jp/) for their permission to use the Chrysanthemum morifolium cultivar 'SeiMarine' and to Ms. Satoko Ohtawa and Ms. Yoshiko Kashiwagi for maintaining the chrysanthemum plants.

\section{Funding}

This work was supported by a research fund of the NARO Institute of Vegetable and Floriculture Science (NIVFS), Tsukuba, Ibaraki, Japan.

\section{Availability of data and materials}

The datasets supporting the conclusions of this article are included within the article (and Additional file 1: Table S1, Additional file 2: Table S2, Additional file 3: Table S3, Additional file 4: Table S4, Additional file 5: Table S5, and Additional file 6: Table S6). Raw sequencing data of chrysanthemum is available with the accession numbers DRR088291-DRR088294, and sequence data of assembled transcripts (contigs) is available with accession numbers IABW01000001-IABW01213204.

\section{Authors' contributions}

KS prepared the CDNA libraries for 454 sequencing. K, Katayose and HK performed the 454 sequencing. AO and K. Kishimoto performed the transcriptome analysis of the metabolic genes. KS, NM, and KN performed the transcriptome analysis of TF genes. NM and KN conducted the bioinformatics analysis. $\mathrm{KS}$ and $\mathrm{AO}$ were the main coordinators of the chrysanthemum EST project. KS was primarily responsible for drafting the manuscript. All authors read and approved the final manuscript.

\section{Ethics approval and consent to participate}

Not applicable.

\section{Consent for publication}

Not applicable.

\section{Competing interests}

The authors declare that they have no competing interests.

\section{Publisher's Note}

Springer Nature remains neutral with regard to jurisdictional claims in published maps and institutional affiliations.

\section{Author details}

'Institute of Vegetable and Floriculture Science, National Agriculture and Food Research Organization (NARO), 2-1 Fujimoto, Tsukuba, Ibaraki 305-0852, Japan. ${ }^{2}$ Plant Gene Regulation Research Group, Bioproduction Research Institute, National Institute of Advanced Industrial Science and Technology (AIST), Central 6, 1-1-1 Higashi, Tsukuba, Ibaraki 305-8566, Japan. ${ }^{3}$ Institute of Fruit Tree and Tea Science, National Agriculture and Food Research Organization (NARO), 2-1 Fujimoto, Tsukuba, Ibaraki 305-8605, Japan. ${ }^{4}$ College of Bioresource Sciences, Nihon University, 1866 Kameino, Fujisawa, Kanagawa 252-0880, Japan. ${ }^{5}$ Institute of Crop Science, National Agriculture and Food Research Organization (NARO), 1-2 Owashi, Tsukuba, Ibaraki 305-8634, Japan

Received: 8 June 2017 Accepted: 14 August 2017

Published online: 04 September 2017

\section{References}

1. Boase MR, Miller R, Deroles SC. Chrysanthemum systematics, genetics, and breeding. In: Janick J, editor. Plant Breeding Reviews, vol 14. Hooken: Wiley; 1997. p. 321-61.

2. Machin B, Scope N. Mutation breeding. In: Chrysanthemum, year-round growing. Dorset: Blandford Press; 1978. p. 34-7.

3. Shibata M. Importance of genetic transformation in ornamental plant breeding. Plant Biotechnol. 2008;25:3-8.

4. Dowrick GJ. The chromosomes of chrysanthemum. II. Garden varieties. Heredity. 1953:7:59-72.

5. Shibata M, Kawata J. Chromosomal variation of recent chrysanthemum cultivars for cut flower. In: Kitamura K, Akihama T. Development of new technology for identification and classification of tree crops and ornamentals. Fruit Tree Research Station, Ministry of Agriculture, Forestry and Fisheries; 1986. p. 41-5.

6. Wang F, Zhang FJ, Chen FD, Fang WM, Teng NJ. Identification of chrysanthemum (Chrysanthemum morifolium) self-incompatibility. Sci World J. 2014; https://doi.org/10.1155/2014/625658. Accessed 21 Feb 2017

7. Stephens LC, Ascher PD, Widmer RE. Interaction among sporophytic S loci in self-incompatible garden chrysanthemums. Euphytica. 1984;33:623-31.

8. Zagorski JS, Ascher PD, Widmer RE. Multigenic self-incompatibility in hexaploid Chrysanthemum. Euphytica. 1983;32:1-7.

9. Arabidopsis Genome Initiative. Analysis of the genome sequence of the flowering plant Arabidopsis thaliana. Nature. 2000;408:796-815.

10. International Rice Genome Sequencing Project. The map-based sequence of the rice genome. Nature. 2005;436:793-800.

11. Langton FA. Chimerical structure and carotenoid inheritance in Chrysanthemum morifolium (Ramat). Euphytica. 1980;29:807-12.

12. Jin JP, Tian F, Yang DC, Meng YQ, Kong L, Luo JC, Gao G. PlantTFDB 4.0: toward a central hub for transcription factors and regulatory interactions in plants. Nucleic Acids Res. 2017;45:D1040-5.

13. Mitsuda N, Ohme-Takagi M. Functional analysis of transcription factors in Arabidopsis. Plant Cell Physiol. 2009;50:1232-48.

14. Coen ES, Meyerowitz EM. The war of the whorls: genetic interactions controlling flower development. Nature. 1991;353:31-7.

15. Ma H. The unfolding drama of flower development: recent results from genetic and molecular analyses. Genes Dev. 1994;8:745-56.

16. Soltis DE. Ma H, Frohlich MW, Soltis PS, Albert VA, Oppenheimer DG, Altman NS, dePamphilis C, Leebens-Mack J. The floral genome: an evolutionary history of gene duplication and shifting patterns of gene expression. Trends Plant Sci. 2007:12:358-67.

17. Colquhoun TA, Clark DG. Unraveling the regulation of floral fragrance biosynthesis. Plant Signal Behav. 2011;6:378-81.

18. Petroni $\mathrm{K}$, Tonelli $\mathrm{C}$. Recent advances on the regulation of anthocyanin synthesis in reproductive organs. Plant Sci. 2011;181:219-29.

19. Preston JC, Hileman LC. Developmental genetics of floral symmetry evolution. Trends Plant Sci. 2009:14:147-54.

20. Kishimoto S, Maoka T, Nakayama M, Ohmiya A. Carotenoid composition in petals of chrysanthemum (Dendranthema grandiflorum (Ramat.) Kitamura). Phytochemistry. 2004;65:2781-7.

21. Nakayama M, Koshioka M, Shibata M, Hiradate S, Sugie H, Yamaguchi M. Identification of cyanidin 3-O-(3,6-O-dimalonyl-beta-glucopyranoside) as a flower pigment of chrysanthemum (Dendranthema Grandiflora). Biosci Biotechnol Biochem. 1997;61:1607-8.

22. Sun H, Zhang T, Fan Q, Qi X, Zhang F, Fang W, Jiang J, Chen F, Chen S. Identification of floral scent in chrysanthemum cultivars and wild relatives by gas chromatography-mass spectrometry. Molecules. 2015;20:5346-59.

23. Woo K, Yu J, Hwang I, Lee Y, Lee C, Yoon H, Lee J, Jeong H. Antioxidative activity of volatile compounds in flower of Chrysanthemum indicum, C. morifolium, and C. zawadskii. J Korean Soc Food Sci Nutr. 2008;37:805-9.

24. Chevreux B, Pfisterer T, Drescher B, Driesel AJ, Müller WE, Wetter T, Suhai S. Using the miraEST assembler for reliable and automated mRNA transcript assembly and SNP detection in sequenced ESTs. Genome Res. 2004;14:1147-59.

25. Mitsuda N, Takiguchi Y, Shikata M, Sage-Ono K, Ono M, Sasaki K, Yamaguchi $H$, Narumi T, Tanaka Y, Sugiyama M, et al. The new FioreDB database provides comprehensive information on plant transcription factors and phenotypes induced by CRES-T in ornamental and model plants. Plant Biotechnol. 2011:28:123-30.

26. Sasaki K, Ohtsubo N. Promotion of efficient molecular breeding using chimeric repressors in ornamental flowers. JARQ. 2016;50:79-86.

27. Bowman JL, Smyth DR, Meyerowitz EM. Genes directing flower development in Arabidopsis. Plant Cell. 1989;1:37-52.

28. Bowman JL, Smyth DR, Meyerowitz EM. Genetic interactions among floral homeotic genes of Arabidopsis. Development. 1991;112:1-20.

29. Sasaki K, Yamaguchi H, Nakayama M, Aida R, Ohtsubo N. Co-modification of class B genes TfDEF and TfGLO in Torenia fournieri Lind. Alters both flower morphology and inflorescence architecture. Plant Mol Biol. 2014;86:319-34.

30. Sommer H, Beltrán JP, Huijser P, Pape H, Lönnig WE, Saedler H, SchwarzSommer Z. Deficiens, a homeotic gene involved in the control of flower morphogenesis in Antirrhinum majus: the protein shows homology to transcription factors. EMBO J. 1990;9:605-13.

31. Tröbner W, Ramirez L, Motte P, Hue I, Huijser P, Lönnig WE, Saedler H, Sommer $H_{\text {, }}$ Schwarz-Sommer Z. GLOBOSA: homeotic gene which interact with DEFICIENS in the control of antirrhinum floral organogenesis. EMBO J. 1992;11:4693-704. 
32. Vandenbussche M, Zethof J, Royaert S, Weterings K, Gerats T. The duplicated B-class heterodimer model: whorl-specific effects and complex genetic interactions in Petunia hybrida flower development. Plant Cell. 2004;16:741-54.

33. Kramer EM, Dorit RL, Irish VF. Molecular evolution of genes controlling petal and stamen development: duplication and divergence within the APETALA3 and PISTILLATA MADS-box gene lineages. Genetics. 1998;149:765-83.

34. Kishimoto S, Ohmiya A. Regulation of carotenoid biosynthesis in petals and leaves of chrysanthemum (Chrysanthemum morifolium). Physiol Plant. 2006;128:436-47.

35. Galpaz N, Ronen G, Khalfa Z, Zamir D, Hirschberg JA. Chromoplast-specific carotenoid biosynthesis pathway is revealed by cloning of the tomato white flower locus. Plant Cell. 2006;18:1947-60.

36. Ohmiya A. Carotenoid cleavage dioxygenases and their apocarotenoid products in plants. Plant Biotechnol. 2009;26:351-8.

37. Tan BC, Joseph LM, Deng WT, Liu L, Li QB, Cline K, McCarty DR. Molecular characterization of the Arabidopsis 9-cis-epoxycarotenoid dioxygenase gene family. Plant J. 2003;35:44-56.

38. Ohmiya A, Kishimoto S, Aida R, Yoshioka S, Sumitomo K. Carotenoid cleavage dioxygenase (CmCCD4a) contributes to white color formation in chrysanthemum petals. Plant Physiol. 2006;142:1193-201.

39. Ohmiya A, Toyoda T, Watanabe H, Emoto K, Hase Y, Yoshioka S. Mechanism behind petal color mutation induced by heavy-ion-beam irradiation of recalcitrant chrysanthemum cultivar. J Japan Soc Hort Sci. 2012;81:269-74.

40. Bouwmeester HJ, Roux C, Lopez-Raez JA, Be'card G. Rhizosphere communication of plants, parasitic plants and AM fungi. Trends Plant Sci. 2007;12:224-30.

41. Dun EA, Brewer PB, Beveridge CA. Strigolactones: discovery of the elusive shoot branching hormone. Trends Plant Sci. 2009;14:364-72.

42. Yoshioka S, Aida R, Yamamizo C, Shibata M, Ohmiya A. The carotenoid cleavage dioxygenase 4 ( $\mathrm{CmCCD} 4 a$ ) gene family encodes a key regulator of petal color mutation in chrysanthemum. Euphytica. 2012;184:377-87.

43. Degenhardt J, Köllner TG, Gershenzon J. Monoterpene and sesquiterpene synthases and the origin of terpene skeletal diversity in plants. Phytochemistry. 2009;70:1621-37.

44. Chen F, Tholl D, Bohlmann J, Pichersky E. The family of terpene synthases in plants: a mid-size family of genes for specialized metabolism that is highly diversified throughout the kingdom. Plant J. 2011;66:212-29.

45. Bohlmann J, Meyer-Gauen G, Croteau R. Plant terpenoid synthases: molecular biology and phylogenetic analysis. Proc Natl Acad Sci U S A. 1998:95:4126-33.

46. Dudareva N, Martin D, Kish CM, Kolosova N, Gorenstein N, Fäldt J, Miller B, Bohlmann J. (E)- $\beta$-ocimene and myrcene synthase genes of floral scent biosynthesis in snapdragon: function and expression of three terpene synthase genes of a new terpene synthase subfamily. Plant Cell. 2003;15:1227-41.

47. Martin DM, Fäldt J, Bohlmann J. Functional characterization of nine Norway spruce TPS genes and evolution of gymnosperm terpene synthases of the TPS-d subfamily. Plant Physiol. 2004;135:1908-27.

48. Yahyaa M, Matsuba Y, Brandt W, Doron-Faigenboim A, Bar E, McClain A Davidovich-Rikanati R, Lewinsohn E, Pichersky E, Ibdah M. Identification, functional characterization, and evolution of terpene synthases from a basal dicot. Plant Physiol. 2015;169:1683-97.

\section{Submit your next manuscript to BioMed Central and we will help you at every step:}

- We accept pre-submission inquiries

- Our selector tool helps you to find the most relevant journal

- We provide round the clock customer support

- Convenient online submission

- Thorough peer review

- Inclusion in PubMed and all major indexing services

- Maximum visibility for your research

Submit your manuscript at www.biomedcentral.com/submit

) Biomed Central 